\title{
The Application of Satellite Simulator in Time Synchronization System Detection in Power System
}

\author{
Zhang Shaohui ${ }^{1, a}$, Sun Fengjie ${ }^{1, b}$, Wang Yang ${ }^{2, c}$, Xing Zechao ${ }^{2, d}$, Teng Ling ${ }^{2, e}$, \\ Gao Qiang ${ }^{2, f}$ \\ ${ }^{1}$ North China Electric Power University,Beijing 102206,China \\ ${ }^{2}$ China Electric Power Reaserch Institute,Beijing 100192,China \\ aemail: 13363001588@163.com, bemail:sfj@ncepu.edu.cn \\ cemail: yangw@epri.sgcc.com.cn \\ demail: xingzechao@foxmail.com ${ }^{\mathrm{e} e m a i l}$ : tengling@epri.sgcc.com.cn \\ femail: gaoqiang2010@epri.sgcc.com.cn
}

Keywords: Satellite Receiver; Satellite Simulator; Time Synchronization Equipment; Time Synchronization Detection

\begin{abstract}
There are many difficulties to establish a real satellite receiver in a laboratory environment. The satellite simulator can replace the satellite receiver to simulate the satellite signals. In this paper, it analyses the advantages of the satellite simulator in the time synchronization detection and consider different time synchronization output signals(1PPS,B code,PTP) as the standard, the difference of the time accuracy is about 10-15 ns according to comparing the satellite receiver with the satellite simulator in the power system time synchronization detection.If taking the margin of error caused by the satellite simulator into account,the satellite simulator can replace the satellite receiver to complete the detection in the power system time synchronization detection.
\end{abstract}

\section{Introduction}

With the rapid development of the power grid, its size and digital level have greatly improved, the performance of network equipments also ask for more stringent requirements, and the lab generate a lot of testing business. Synchronous electrical equipments in the laboratory should follow a high-precision time or frequency reference, this reference is usually provided by a satellite signal. However, using real satellite receiver in a lab environment is very difficult. For example, the changes of experimental location,the non-reproducibility of real satellite signals, etc. Thereby the synchronization system detection were impeded.

Satellite simulator can simulate the output of different environments and different geographic locations, it can replace the real satellite signal in principle. The article use theoretical analysis method, respectively, take different time synchronization output signal (1PPS, B code, and PTP) as the reference to explore whether the satellite simulator can replace the satellite receiver to complete the detection of time synchronization system in the power system.

\section{Satellite Receiver}

Satellite receiver is the instrument that receiving satellite signals [1], it get the local position information and time information according to the acquisition and demodulation of satellite signal, Satellite signal demodulated is widely used in various fields.

Satellite receiver can be divided into two kinds,the first one is dynamic receiver, the other one is static receiver. Dynamic receiver is set up in the mobile carrier. Static receiver is mainly used in a lab environment. Consisting of satellite receiving antenna is shown in Figure 1. 


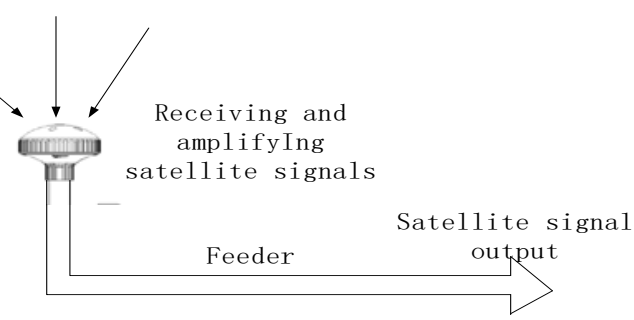

Fig.1 The system components of satellite receiving antenna

\section{Satellite Simulator}

Satellite simulator is the equipment that use the simulation software to simulate the satellite signal. Satellite simulator is made of software simulation host computer and digital signal generator.

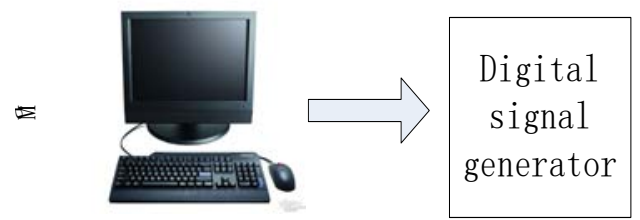

Fig.2 The system components of satellite simulator

Software simulation host computer is to use an external computer and simulation software to simulate various parameters[2] satellite signals, and set the time of the satellite signal simulation coordinates, ionospheric delay, the carrier state of motion parameters and interference types according to the needs of users. After the user setting the desired satellite signal parameters, simulation software build the navigation message correction value model parameters and output analog satellite signal and sends the signal to the digital signal generator.

Digital signal generator receives the parameters of analog satellite signal and then spread process, add the Doppler effect, after this, it simulate the digital baseband signal of real environment.

Depending on the number of different channel satellite simulator will be divided into two kinds of single and multi-channel, multi-channel simulators typically have 12 channels; It also can be divided into single-mode satellite simulator, dual-mode and multi-mode satellite simulator according to the number of different types of satellite signals; If the motion status signals have special requirements the Satellite simulator can be divided into a high dynamic, low dynamic satellite simulator.

\section{Comparison of Satellite Receivers and Satellite Simulator}

Satellite simulator simulate the entire work process according to the space satellites ,orbit and then link users,it also consider the link error, the earth's rotation and Doppler effect.

Comparing to the satellite receivers, the satellite simulator still has obvious advantages in experimental tests:

1.Reproducible test. The researchers could conduct experiments repeatly under the same test conditions, thus improving the accuracy.

2.The accuracy of the test. Satellite simulator could adjust the power and various other parameters of satellite signal in order to meet the needs of different experiments[4].

3.Customizable scene. It can simulate different weather conditions, atmospheric parameters according to the experiment needs.

Satellites in orbit have the 10-13 accuracy of high-precision time reference[5]. The emitted satellite signals own a high quality time level. Satellite simulator itself has a crystal clock that can offer the time accuracy of 10-9, although inferior to the accuracy of real time signal, but still meet most laboratory testing needs. If you need more accurate time reference, the simulator can be configured with a external clock reference source which have frequency output with high accuracy. 


\section{Comparison of Satellite Simulator and SatelliteReceiver for Power System Time Synchronization Detection}

In the power system, the detection method of device for time synchronization performance is shown in Figure 3.

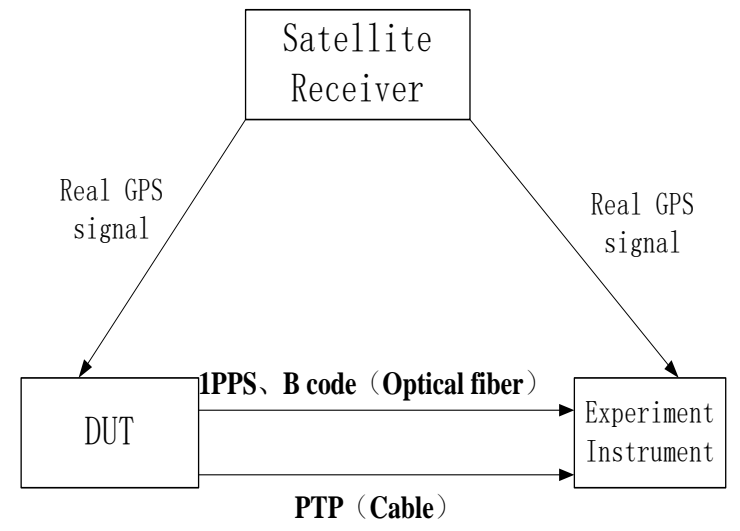

Fig.3 The time synchronization detection performance of power equipments

Satellite receiver between the DUT and the test instrumentation are connected with feeder to transmit satellite signals, between the DUT and the test instrument are connected with optical fiber or cable to deliver different types of time synchronization output signal(1PPS, B code and PTP). Test equipment and test instruments are timed with homologous satellite signal in order to achieve the same time reference. Experiment instrument compare the signal received from the DUT with its own reference, the time difference between the two signals is the time accuracy of the device under test.

Article will take the GPS satellite signals produced by the satellite receiver and satellite simulator as the time reference base, and use the 1PPS, B code, and PTP as the time synchronization output signal to test the synchronization performance of the equipment.According to the results, we can explore whether the satellite simulator can replace the satellite receiver to complete the detection of time synchronization system in the power system.

\section{(1)Satellite Receiver as Time Reference}

The principle of taking the real GPS signal as reference in shown in Figure 3. Respectively, different time synchronization output signals are used to detect time synchronization device performance.

\section{1) 1PPS}

1PPS is called second pulse signal, the signal does not contain the time information, the start of the rising edge of signal per second is the the exact time.It is usually used in the tests, its time accuracy is 100ns. Between the DUT and the test instrumentation using optical fiber to transmit 1PPS signal.

Based on the above experimental program, 12 hours test time, 43200 sampling points, and obtain an average error of $81.30 \mathrm{~ns}$.

To ensure the accuracy of the results, three sets of experiments are carried out under the same conditions and find the average number of three groups. The average error is $81.68 \mathrm{~ns}$.

\section{2) B Code}

Between the DUT and the test instrumentation using optical fiber to transmit B code.

Similar with 1PPS, 12 hours test time, 43200 sampling points, and obtain an average error of 77.66 ns. Taking the average of three experiments, the results is $77.86 \mathrm{~ns}$.

\section{3) PTP}

PTP signal use the data network to transfer time synchronization information and it has high precision. Between the DUT and the test instrumentation using cable to transmit PTP signal.

Similar with the two tests above, 12 hours test time, 43200 sampling points, and obtain an average error of133.56 ns. Taking the average of three experiments, the results is $134.50 \mathrm{~ns}$. 
Similar with the satellite receiver, replace the satellite receiver with satellite simulator and repeat the test. Final results are shown in Table 1

Tab.1 The results when simulated GPS signal is the reference

\begin{tabular}{cccc}
\hline Signal Type & 1PPS & B Code & PTP \\
\hline Time Accuracy & $67.65 \mathrm{~ns}$ & $67.29 \mathrm{~ns}$ & $119.80 \mathrm{~ns}$ \\
\hline
\end{tabular}

\section{(3) Analysis of Results}

All the above experimental results are shown in Table 2

Tab.2 Experimental results and electricity standards

\begin{tabular}{cccc}
\hline Signal Type & Power Standard & $\begin{array}{c}\text { Real GPS Signal is the } \\
\text { Reference }\end{array}$ & $\begin{array}{c}\text { Simulated GPS Signal is the } \\
\text { Reference }\end{array}$ \\
\hline 1PPS & $1 \mu \mathrm{s}$ & $81.30 \mathrm{~ns}$ & $67.65 \mathrm{~ns}$ \\
B code & $1 \mu \mathrm{s}$ & $77.86 \mathrm{~ns}$ & $67.29 \mathrm{~ns}$ \\
PTP & -- & $134.50 \mathrm{~ns}$ & $119.80 \mathrm{~ns}$ \\
\hline
\end{tabular}

When the real GPS signal is the reference[6,7,8], the result is about $80 \sim 130 \mathrm{~ns}$, and the result under the same the same situation of simulated GPS signal is 10 15 ns than the real GPS signal. The error percentage is about $11 \% \sim 18 \%$,so in a lab environment we can selectively use a satellite simulator instead of using a satellite receiver for testing. In the relative measurement experiment, satellite simulator can be directly used for all types of timing. In the absolute measurement experiment, we recommend to consider the introduction of satellite simulator error and correct the results.

\section{Summary}

The article takes the 1PPS, B code, and PTP as the output signal, according to the comparison between the satellite receiver and satellite simulator in the tests, the conclusion is: In the time synchronization detection of power system, if considering the reasonable error, the satellite simulator can replace the satellite receiver to complete the detection.

\section{Acknowledgement}

In this paper, the research was sponsored by the China State Grid Corp technology project: the key technology research and application of the power synchronization key technology based on the time frequency fusion

\section{Reference}

[1] Wang Yu Dong, Yu Tian Qing. The research on communication power system time synchronization network [J]. The communication of electric power system, 2009,30 (7): 64-67

[2] Chen Bao Ren. Research on the construction scheme of the power network integrated [J]. Power Survey and design, 2012, (2): 57-62.

[3] Liu Li Na. The development of power system communication integrated network management system [J]. power system communications, 2004,25 (7): 5-7

[4] Wang Wu Chao. Power integrated network management system for communication network construction of [J]. power system communication, 2006,27 (5): 16-20

[5] Liu Tong Na. The power communication network management protocol [D]. North China Electric Power University (Hebei), 2003

[6] Li Tian Jian, Zeng Wen Fang. Analysis and Prospect of application of [J]. network management 
protocol of the computer system, 1999,05:17-20.

[7] Liao Jian Xin, Zhang, Wang Jing, Meng Hui Yang. Mobile intelligent network management system analysis and design [J]. Modern telecommunication technology, 2002,05:22-24+41.

[8] Lu Yan, Peng to offer. Research and application of CORBA in the network management [J]. modern electronic technology, 2006,10:47-49. 